\title{
The Impact of Socioeconomic Variables Status on Bladder Cancer Treatment Outcomes during the COVID-19 Pandemic
}

\author{
Chamseddine Barki ${ }^{*}$, Hanene Boussi Rahmouni1,2, Salam Labidi1 \\ ${ }^{1}$ Research Laboratory of Biophysics and Medical Technologies, The Higher Institute of Medical Technologies of Tunis, \\ Department of Biophysics, University of Tunis El Manar, Tunis, Tunisia \\ ${ }^{2}$ The Computer Science Research Centre, University of the West of England, Bristol, UK \\ Email: *chamseddine.barki@istmt.utm.tn
}

How to cite this paper: Barki, C., Rahmouni, H.B. and Labidi, S. (2021) The Impact of Socioeconomic Variables Status on Bladder Cancer Treatment Outcomes during the COVID-19 Pandemic. Open Access Library Journal, 8: e7921.

https://doi.org/10.4236/oalib.1107921

Received: September 2, 2021

Accepted: September 23, 2021

Published: September 26, 2021

Copyright () 2021 by author(s) and Open Access Library Inc.

This work is licensed under the Creative Commons Attribution International License (CC BY 4.0).

http://creativecommons.org/licenses/by/4.0/

\begin{abstract}
Social inequalities are concerning along the bladder cancer (BC) continuum especially within the malignancy outcomes and treatment effects and complications. Moreover, the COVID-19 crisis and its variants have uncovered major inequalities between communities and added more pressure on socioeconomic (SE) status along with the COVID-19-associated variants. Within this global situation, discerning the societal risk factors that render specific communities vulnerable is crucial to ensure more effective management of $\mathrm{BC}$ malignancy. In this paper, we aimed to investigate the status and the impact of SE variables and disparities on BC survival as reported in recent studies and give an update on the impact of social environment on treatment outcomes within COVID-19 pandemic. Several studies showed that SE status is a significant predictor of survival for BC patients. Recent evidence found that $\mathrm{BC}$ outcomes are directly affected by social deprivation. Our results confirm that there are major and recurrent SE disparities in BC survival. Together, the findings demonstrate the considerable impact in public data-based centers, albeit reportings are partial and inaccurate, of SE and health inequities in severely segregated neighborhoods under the weight of the COVID-19. The findings show the major influence of healthcare inequalities SE deprivation in a distinctly segregated community within the COVID-19 burden and death in centers according to public statistics, even if reports are unfinished and partial.
\end{abstract}

\section{Subject Areas}

Economics, Epidemiology, Global Health, Sociology 


\section{Keywords}

Bladder Cancer, Complications, COVID-19, Public-Health, Socioeconomic Status, Treatment Outcomes

\section{Introduction}

Even though global health indicators of bladder cancer (BC) treatment change often in response to socioeconomic (SE) factors such as the patient's economic and social status, a social gradient of premature mortality persists across the world, including developed countries [1] [2]. A statistically significant incidence gradient increasing with social deprivation was observed for $\mathrm{BC}$ in both sexes. Its incidence is steadily increasing across the world. $\mathrm{BC}$ is one of the pathologies that contribute the most to this SE gradient and reducing health inequalities is a prime concern [3].

Although fighting health inequalities has been a priority for international health organizations for many years, their focus on the agenda of health and social policy is still new, as evidenced by recent public and global health laws and missions entrusted to regional, national, and international health agencies [4]. $\mathrm{BC}$ remains a significant public health concern. The standardization of clinical practices and therapeutic approaches to combat $\mathrm{BC}$ serves as the foundation and point of reference for patient triage and personalized care [5]. As a result, by incorporating the patient's preferences into the therapeutic decision-making process for his disease, the treatment outcomes are significantly improved [6]. Furthermore, many problems have emerged since the economic and social global health crisis caused by the COVID-19 pandemic [7]. To ensure that the benefit of early intervention for $\mathrm{BC}$ outweighs treatment side effects combined with the risk of contracting COVID-19 in the healthcare environment, clinicians and patients must participate in a joint decision-making [8]. On the other hand, due to SE constraints that impose and affect the effects of the prescribed treatments and, as a result, the expected outcomes, the patient's, and treatment's choice are not always what is desired. The expected outcomes are primarily the treatment's effect and adverse events, which are then conditioned by the decision made on the basis of the SE impact of improving or worsening the situation [9]. This has an indirect effect on the rate of survival and mortality, making it a public health concern. According to a recent review of data from the International Agency for Research on Cancer (IARC), estimating that the global cancer burden has increased to 19.3 million cases and 10 million cancer deaths in 2020, as reported on December $14^{\text {th }}[10]$. Non-muscle invasive bladder cancer (NMIBC) represents $65 \%-75 \%$ of the detected cases, however, muscle invasive bladder cancer (MIBC) represents $20 \%-30 \%$ [11]. Moreover, it was claimed that one in every five people gets cancer at some point in their lives, and one in every eight men and one in every eleven women dies from it. According to these recent figures, 
more than 50 million people are alive within five years of a previous cancer diagnosis [12]. Globally, aging demographics and SE risk factors continue to be the key drivers of this rise. According to the new global cancer observatory (GCO) database, the estimated number of worldwide patients diagnosed with $\mathrm{BC}$ is 573,278 people, making $\mathrm{BC}$ the tenth most widely diagnosed cancer on the planet [13] [14]. Statistics indicate that $4.4 \%$ of the total new detected cases (440,864 new cases around the world) were diagnosed in men which make BC, the sixth most frequently diagnosed cancer in men around the world [15]. Based on the latest 5-year prevalence results, 1,720,625 BC patients are living five years after a previous diagnosis [16].

While $\mathrm{BC}$ has distinct demographic characteristics, the impact of the SE status and factors such as age, gender, race, and ethnicity on the results of the disease is not well known and poorly explained. There are few thorough inquiries into these topics, with social inequality possibly attracting the least attention as studies made by Ahmadi et al., Shackley et al., Yee et al. and Haque et al., which needed further study of SE variables status, access and quality of care and treatment decision making among $\mathrm{BC}$ patients to better explain the impact and manage these disparities. [17] [18] [19] [20]. The present and ongoing literature and epidemiological research on the impact of SE status on the outcome of BC are reviewed in this paper.

To ensure more effective care of $\mathrm{BC}$ malignancy in this worldwide environment, it is critical to identify the SE risk factors that make individual groups vulnerable. The goal of this research was to look at the current state and influence of SE determinants and disparities on BC survival as reported in recent studies, as well as provide an update on the role of the social environment on treatment results in the COVID-19 pandemic. Moreover, we aimed to look at new evidence about how social deprivation affects $\mathrm{BC}$ outcomes and the prevalence of SE disparities in BC survival.

\section{Materials and Methods}

SE status can be measured using various methods of different features. Special indexes including social class, domiciles or living quarters, occupation or employment status, wage, transport information and personal living status are commonly used in the recent European and American studies [21]. Moreover, indexes based on education and geographical areas indicated by the zip codes of patients were lately included in the studies [22]. To create a SE status ranking for a given region, these indexes merge several factors, most of which are derived from national census data. The underprivileged area score (UAS) as identified by Jarman et al. [23], the material and social deprivation score as described by Townsend et al. [24], the classification SE confounding index used in spatial epidemiology as developed by Carstairs et al. [25], and the index of multiple deprivation (IMD) according to Abel et al. [26] and the European deprivation index (EDI) as reported by Launoy et al. [27], are all common methods for esti- 
mating deprivation defined as indexes for measuring SE disparities between areas. Commonly, data is divided into quintiles for an entire area, resulting in the five SE status stages between the least (Q1) and the most (Q5) deprived quintile [28]. The home zone will be assigned to one of these quintiles and will be ranked accordingly. Obviously, using such area-based indicators stands for personal patient SE status. Thus, when people with high SE status live in disadvantaged areas or the inverse, confusions about estimations with possible inaccuracies can happen. In spite of the diversity of SE status indexes used worldwide, it has been shown that they have a tendency to be coherent in healthcare research studies to reach a common level of agreement [29].

\section{Results}

\subsection{The Impact of Socioeconomic Status Disparities on Cancer Survival and Mortality Rates}

Several research studies indicate a strong direct relationship between premature death, comorbidity, and the poor SE conditions [30] [31] [32]. A wide comprehensive search was made using keywords and domain terms. We used references to extend and collect more knowledge about the topic. Within a systematic review, we included the most relevant studies from which we extracted the most appropriate outcomes. A patient's demographic circumstances or SE status may influence the disease's prognosis, especially within the COVID-19 crisis. Several observational findings in recent years have found that low SE status may have a negative impact on cancer patient survival [33] [34]. In a study directed by Kuhn et al., results showed that social conditions as well as SE status influence COVID-19-cancer patients' survival and similar impacts of SE status on cancer survival have been identified in other studies [35]. While inequalities in cancer survival can be best correlated with indexes specific to patient's SE status, aggregated indicators data may help understand the impact of the social factors.

On the other hand, SE status can impact the cancer survival indirectly, through a variety of mediating indexes: Low-wage patients do not have convenient and available access to healthcare services that will let them benefit from an early clinical assessment or a high-quality treatment with a high level of efficiency and low risk of complication. Many experiments used mediation assessment to explore the indirect impact and the implication of a mediator index. In their studies, Russell et al. [36], Luo et al. [37], and Richiardi et al. [38], found that the impact of the SE status on patient survival time can be identified by mediator indexes involving patient's life and work ecosystem, cancer treatment type namely radiation therapy [39] and immunotherapy, cancer treatment possible side effects or further malignancy risk factors including demographics.

According to a recent study carried out by Bryere et al., the SE environment is strongly associated with the risk of developing certain cancers [40].

The published data made it possible to measure the strength of this correlation. This could be explained by the inequality of access to healthcare services, 
the occurrence of cancers which are often more severe within the most disadvantaged populations, or even by other health problems. A prospective French observational study explained this fact, in which a total of $5331 \mathrm{BC}$ patients over 109,071 men diagnosed with cancer cases were observed closely for 4 years [41]. Almost $58 \%$ of the cases are dead, and COVID-19 variant D empowered this rate. Moreover, they reported that the most death percentage is found in the Q5 most deprived quintile with $23.4 \%$ compared to $16.5 \%$ in Q1 as the least deprived quintile with 3 years medium follow-up time and the detected median age is 73 years. The study published by Guy et al., using data from the Francim network, provides more details about the impact of SE status on the risk of occurrence of different cancer locations and post-therapy survival rates within the prescribed or the received treatments [42].

The Caen researchers looked at the most frequent 18 different cancers, using the Francim network, to analyze a population of 189,144 cancer cases [43]. For each patient, the status of social deprivation was assigned using an index calculated with reference to the place of residence. By comparing the incidence of the considered cancers and the deprivation index, several important results emerged: Statistically, BC and cancers of the stomach, liver, ENT (Ear, Nose, Throat) and lung in both sexes; cancers of the esophagus, pancreas in men; and cervical cancers in women, occur more frequently in socio-economically disadvantaged populations (Q5). Conversely, melanoma in both sexes, prostate and testicular cancers in men, and ovarian and breast cancer in women are more common in more advantaged populations (Q1). Thus, results estimating the impact of the SE factor showed how crucial it is to improve the living conditions and promote the health within the most disadvantaged populations: around $25 \%$ of $\mathrm{BC}$ and laryngeal cancer cases are thought to be attributable to social deprivation, 27\% of cases of ENT cancer, 20\% of lung cancer cases and almost 15,000 cases of cancer in total according to the study.

Cancer stage and extension in time and space have been identified as the most significant predictive factors, and many studies have examined and confirmed the close relationship between the SE status, the stage of cancer and survival rate [44]. Considering the physical loss and damages caused by COVID-19, in tests on $\mathrm{BC}$ and other types of cancer such as prostate, breast, kidney, skin and stomach, patients with lower SE status typically have a more advanced cancer stage at diagnosis with less treatment effectiveness and more adverse events. This was shown in results indicating that patients with a lower SE status are more likely to experience more serious complications, as reported in the studies of Golombos et al., Sun Seog et al., Sung et al. and Mihor et al., [45] [46] [47] [48]. This research emphasized the importance of stage as a significant predictor associated with SE status in terms of survival. Hence, the indirect impacts of SE status on cancer condition involve the required mechanisms that need to be investigated further. Besides, healthcare researchers and providers must consider the causal process that links a planned management change to a possible effect on patient 
safety in order to predict the effects of a procedure or treatment protocol that has not yet been implemented [49]. Assessing the direct or indirect impact of social and demographic indexes on survival time applying a decomposition approach will include the high-quality evidence needed to make management decisions regarding the distribution of health care services.

Furthermore, previous studies were able to associate the risk of cancer mortality and the cancer survival with occupational class disparities and a poor SE status. Lee et al., found that, within Korean men of different occupational classes observed for 13 years, men in manual labor and compensation by an hourly wage occupation had lower survival of cancer than men who worked in technical and administrative roles which had [50]. The manual labor class had a death rate adjusted for age and year of diagnosis/treatment of 1.48 [95\% confidence interval: 1.45 - 1.51] compared to the higher occupational classes. Tumors with a favorable prognosis, such as the urinary tract cancer, demonstrated a greater disparity throughout professional classes. Among Korean citizens, there were more significant differences in cancer survival based on profession within the COVID-19.

Cancer prevention policies should place a greater emphasis on the lower SE occupational community. In another study carried out by Silvia et al., observations about a study involving British men of different SE positions for 24 years, found that men with a low SE position had higher cancer mortality [51]. The lowest SE position men had a death rate adjusted for age of 1.07 [95\% confidence interval: 0.76 - 1.52] compared to the highest SE position. A relationship between SE status and mortality was significantly explained by adjusting for health behaviors, particularly when the behaviors were tested repeatedly. Evidence built on results and conclusions report that patients from higher SE backgrounds had greater survival rates across a large spectrum of cancers [52] [53].

Nina et al. carried out a study about trends and SE disparities in cancer survival by SE deprivation at the local level [54]. In their study, they targeted a population of 331,419 Victorian people aged 15-99 and diagnosed with cancer between 2001 and 2015. It showed that cancer survival depended on SES (as evaluated by Socio-Economic Indexes for Areas, SEIFA, Index of Relative Socio-economic Disadvantage (IRSD)/quintiles). A low IRSD score refers to a high proportion of economically and socially deprived individuals in a community. Patients of the most deprived communities have less survival rates with a 5-year relative excess mortality rate ratio 1.40 [95\% confidence interval: 1.32 - 1.49] compared to patients of fewer disadvantaged areas. Particularly, the disparities in survival were generally larger in younger patients under 55 years old within BC, lately devasted by the COVID-19 virus.

Although cancer survival has increased in recent years, there has been a widening unavailability disparity, particularly after the great financial recession, which was an acute fall in economic activity and was the most serious economic slowdown since the great depression in the late 2000s [55]. It has been reported that the survival rate increases for patients who live in comfortable areas com- 
pared to those who live in impoverished areas. This troubling note has already been observed as well as in the developed countries, even after improving the growing disparities in the global mortality rate between social classes [56] [57].

\subsection{Socioeconomic Status Factors Influencing Cancer and Treatment Outcomes}

Several alternative explanations and causes have been proposed for the lower survival rate found in patients diagnosed and treated for cancer from more deprived areas. Studies showed that lower social classes with poor income get access to the healthcare system to benefit from medical services less than their need would really suggest [58]. Adding to that, other studies found that some patients of the Q5 most deprived quintile category seek unnecessary services and may not target the proper destination which makes them waste time, money, and efforts. When investigating this issue within COVID-19 crisis, they reported that the lack of financial resources is one the reasons pushing them for example to buy medicines and drugs randomly without medical prescription or consult a general practitioner rather than finding a specialist so that they can spend less money [59] [60]. This complicated the cancer treatment workflows and extended therapies duration and more side effects including the highest risk grade of mortality appeared especially within urinary tract cancers. Access limitation factors may be related to a lack of knowledge and evidence about the disease and its treatments, time and place issues, patient's level of consciousness and awareness about cancer therapy and possible side effects or adverse events. Generally, even when symptoms appear, these patients prefer to postpone their medical visits and their access to healthcare services later compared to patients within higher SE status who are more vigilant, available, and clinically interactive [61].

The deprivation gradients identified for all-cause survival of $\mathrm{BC}$ and COVID-19 infection have also been proposed to act as a key-index that reflects a non-cancer-related death. There have been several studies investigating correlations between or adapting to suffering and such causes [62].

Patients in rural areas suffer from higher death rates due to poverty, inefficient health risk management, less immunization actions, and poor prognosis with low screening frequency compared to urban areas, in line with the widespread gap in survival and lifetime expectations between rural and urban regions.

Vanthomme et al., found that, in under-secondary men aged 40 - 64 the mortality rate is about 167 per 100.000 person-years [95\% confidence interval: 164-171] for lung cancer deaths. This number has increased almost 2.4 times compared to tertiary education men with 68 per 100,000 person-years [ $95 \%$ confidence interval: 65-72]. For older patients, the death risk among the under-school men is about 3.3 times higher 566 per 100,000 person-years [95\% confidence interval: 558-575]. Higher educated patients have a protective effect on cancer mortality risk thanks to their behavioral and SE resources [63]. SE status is an important factor and a major index to identify and predict cancer 
mortality within patients. An estimation presented by Siegel et al., mentioned that one fifth of all deaths would not happen if patients were affiliated to tertiary education according to the American cancer society epidemiologists [64] [65]. Despite factors related to ethnicity and race, the survival of cancer is well witness and highly perceived within patient of a better SE status [66].

However, SE trends have shifted significantly in terms of cancer mortality within the COVID-19. Many years ago, the SE condition was highly linked to carcinogenicity rates, which showed a higher risk of cancer death among the prosperous population [67]. In the late years, this association has gone the other way, with wealthier patients having a less chance to die from cancer than other patients with private and special health care thanks to the improvements in preventive care, early cancer diagnosis and cancer treatments.

Previously, death from urinary tract or prostate cancers did not change significantly in SE condition. Now, there is an inverse SE gradient [68] [69]. This inequality is identified as a lead cause for the over mortality of people living in the impoverished regions, due to cancer [70].

SE impoverishment of the environs can also be associated with shorter telomere length, fatal cancer sign and a premature aging [71]. In most cancer types, cancer disparities and death rates are obvious and show SE imbalance and considerable risk factor vulnerability variations [72]. Emerging research has raised concerns about the disproportionate impact of COVID-19 morbidity and mortality among racial and ethnic minorities, which has been linked to SE factors that put low-income people at higher risk of infection. The impact of racial and SE characteristics on COVID-19 transmission and illness severity is yet unknown and needs to be investigated further. The rise in COVID-19 patient volume resulted in more missing data on key covariates such smoking status and race.

\subsection{COVID-19 and Socioeconomic Disparities within Bladder Cancer Patients}

The widespread of the COVID-19 infectious disease caused by the SARS-CoV-2 virus, had a devasting impact on already established cancer care disparities [73]. Moreover, the pandemic caused significant delays in cancer treatments and led to an unexpected social upheaval. Emerging studies reveal that the COVID-19 pandemic can increase social inequities, particularly in cancer patients. In a study carried out by Kamal et al. on 18,650 patients with both COVID-19 and cancer found that the risk of death is considerable for cancer patients with COVID-19 [74]. Researchers reported that $22 \%$ of this population died with a death probability of $25.6 \%$ [ $95 \%$ confidence interval: $22.0 \%-29.5 \%$ ]. Access to healthcare platforms, treatments, telemedicine, quick diagnosis, and availability to therapy may be potential factors of unequal survival of the pandemic cancer. Although the cancer guidelines proposed by oncology society during a pandemic are greatly impacted by the extent of prospective treatment advantages, treatment intention and availability to care and the prioritization of delivery of can- 
cer medicines [75].

Even more, SARS-CoV-2 variants appeared in high populated cities and countries with deprived quintile and many observations mentioned that this disease when associated to SE inequalities made it possible for other diseases to appear and be more dangerous such as cancers and Mucormycosis (black fungus) to use them as deadly weapons and increase mortality rates in this social category. The Indian government declared that more than 8800 COVID-19 cases died with Mucormycosis rare infection [76]. According to the Centers for Disease Control and Prevention (CDC), the B.1.617 Indian variant of COVID-19 increased black fungus infection probability with a death rate of 54\% [77]. Scientists found that the use of steroids for COVID-19 treatment may be one these causes [78]. This derived infection is life-threatening in cancer patients at the first place. The treatment is very expensive and up to death rate may reach $90 \%$ as expected in India within the coming days, especially with the SE disparities and the extended deprived quintile areas, according to local physicians. Strong departmental disparities are always seen according to every single variant. It has been progressively obvious that the densely populated cities are most affected by this disease, since the first reported case of COVID-19 [79]. Although COVID-19 has had widespread influence, the revelation of SE status layered statistics has validated worries over its disproportionate influence on minority communities. The causes have been extensively examined, including medical history illnesses, living circumstances and healthcare status.

Cancerologists and oncologists had to make tough judgments to weigh up the advantages of treatment and deal with hazards of infection with COVID-19 and the high possibility of mortality for cancer patients. Chemotherapy, surgery, and radiation regimens have been postponed or adjusted to speed up treatment and reduce risks of coronavirus infection. According to a study carried out by Dai et al., among patients COVID-19 patients with cancer, over 33\% death rate was witnessed within patients receiving immunotherapy and $25 \%$ for those who underwent surgery procedures [80].

The COVID-19 pandemic will worsen existing cancer differences in deprived areas and will determine diverse paths for neighborhood cancer patients through the SE partition and disparities. Worries are predicated on the fact that minorities are more susceptible to engage in important manual labor without having the chance to work from home. The requirement to work outside in this global crisis, raises the danger of SARS-CoV-2 infection risk and death. Furthermore, this category of people is at a high probability to have low-paying occupations that offer minimum or no medical insurance care.

Patients who need to leave their houses and be present at the workplace during the pandemic are at danger of contracting the virus and may suffer from cancer care delays, as well as the uninsured patients who may lose their employment and health during the pandemic.

The implication of Telemedicine has been enhanced by the present health crisis but limitations and difficulties in using technology, have left deprived pa- 
tients at danger of fragmented treatment and loss of care. Several observations reported that patients with less education and lower SE status are with less intentions to be involved in telemedical services [81] [82]. Moreover, low knowledge rates on health, limits effective advocacy by patients. All these variables and circumstances will probably exacerbate their cancer results.

Although infectious disease is causing the present health crisis, the rising infection rates among cancer patients raises the chance of get COVID-19, leading to treatment delays and serious complications.

\subsection{Knowledge-Based Bladder Cancer Outcomes and Socioeconomic Impacts}

The main recent studies assessing the results of BC related to SE differentiated deprivation have been found in the literature. All of them were directly linked to poor survival and low SE status.

Russel et al. [83], have studied the link between BC survival and SE deprivation by discovering possible mediation agents for relationships in the survival of BC patients with a differing SE level. Based on the BC Database in Sweden, more than 37,000 people were diagnosed with Tis and Ta-T4 BC as observed closely for 17 years. Educational degree was employed as a SES substitute. In order to study the relationship between SES and survival, accelerated failure time models were used. Observations showed that almost $50 \%$ of patients had low SE status levels, 35\% with a moderate SE status level, and around 15\% with a high SE status level. To study the interaction mediators of the relation, mediation analysis was assessed. The overall and BC-specific survival of patients with high SE status was at a rise in this research compared to individuals with low SE status. The total survival of the first quintile NMIBC patients was 1.93 [95\% confidence interval: 1.82 - 2.05] and for MIBC it was 2.14 [95\% confidence interval: 1.92 - 2.39] compared to 1.00 [95\% confidence interval] for both types of patients for quintile five. In patients with NMIBC, the Charlson Comorbidity Index mediated $10 \%$ of this association and $4 \%$ of the hospital type. In MIBC patients, the period after the reference to TURBT was a significant mediator $14 \%$. Even after making adjustment to independent pronouncements (age, CCI, marital status, hospital, clinical $\mathrm{N}$ phase, clinical $\mathrm{M}$ stage, and WHO class). This conclusion was kept accurate. The researchers observed that many factors might explain the relationship between SE status and BC survival. Nevertheless, the mediators revealed could not explain the fully proposed causal relationships. These results emphasize the necessity of healthcare uniformity within SE categories. The risk to BC was high with low treatment outcomes to smokers of fifth quintile (19.5\%) compared to the first quintile smoker patients (12\%). This work is crucial since it has particularly looked at causal relationship between death/survival and SE status.

Although many cancer tumors show a relationship between poor SE status and lower survival rates, there were some research that reported survival results related to SE status and BC. An American research team carried out a study to 
identify the differences in advanced BC diagnosis and treatments [84]. Researchers detected inequalities in overall survival, therapy, and time for treatment among the patients. Patients diagnosed with urothelial BC, were selected, and extracted from the National Cancer Database. The relationship between variables and prognosis of the cancer was evaluated using multivariable logistic regression. In this investigation, Cox proportional tests, log-rank and Kaplan-Meier were also applied to assess inequalities of overall survival. Treatment and processing delays between subgroups were also studied.

The study revealed that lower levels in education and incomes were linked to a high risk of progressive illness and poorer overall survival. Patients of the fourth quartile also were less treated within the first months of being diagnosed with MIBC. Of the selected 328,560 individuals, almost $8 \%$ were found with and advanced $\mathrm{BC}$. The elevated probabilities of severe illness were linked to women more than men, race, and regions with lower incomes. The overall survival of women was poorer than men 1.16 [95\% confidence interval: $1.12-1.20$ ]. Moreover, it was lower for the fourth quartile regions 1.08 [95\% confidence interval: 1.02 - 1.16]. The increased survival was found within chemotherapy and radical cystectomy 0.55 [95\% confidence interval: $0.53-0.57$ ] and radical cystectomy 0.61 [95\% confidence interval: 0.59 - 0.64]. According to gender, race and lower region incomes, therapy was lower in time and quality. Patients with quartile of low levels of education and income had considerably increased delayed care and treatment $16 \%$ compared to those with first quartile levels $12 \%$. Patients with no insurance were at a high rate of time to treatment delays 13\% than insured patients. Prognosis and therapy of $\mathrm{BC}$ are subjects to significant differences.

Another study carried out by David et al., investigated if SE status is a risk factor for post-treatment adverse events and oncological results following BC therapy [85]. In $383 \mathrm{BC}$ patients who received the treatment in a tertiary referral facility, a retrospective examination was carried out for the period of 13 years. In addition to clinical and pathological indicators for risk classification, prospective social health factors were assessed. The SE status using region indexes included average yearly income and educational degrees. Based on SE factors, patients were assessed on possible disparities. In order to find the 3 months post-treatment adverse events predictors, multivariate logistic regression was then employed. Results showed that more than $45 \%$ of patients developed problems and significant predictors of side effects were: Income, duration of stay, lower revenue group and lowest education grades. In terms of education as well as income there have been no big variations in the overall survival estimates. With a low income and low education level, post-treatment complications were separately combined, but no influence was identified on overall survival for 37 months. The likelihood of perioperative problems is within patients with lower SE level undergoing BC treatments. The rate of patients with side effects has raised from $11.2 \%$ in the least deprived quintiles to $16.1 \%$ in the most deprived after the identification of SE deprivation ratings. For patients within the most deprived quartile, the 
probability of major complications was two times higher [86]. It is of a high importance to ensure the communication with patients, motivation and adherence to post-treatment follow-up and support systems. The authors claim that the effect may have minimized the influence on oncological results for patients being carefully attended at a large tertiary facility. Azadeh et al. created a measuring index of SE status and compared outcomes of all BC patients on which it was based besides the insurance status. Even when tumor characteristics were controlled, low SE status with no insurance was associated with worse overall survival [87].

\subsection{Bladder Cancer Treatment Effects and Costs}

Recently, substantial progress in new treatments for BC types has been made with reference to the efficacy and side effects constraints. Diagnostic and staging tests, targeted treatments, immunotherapies, and a variety of local innovations and techniques have all been linked to increased patient lifespan and quality of life [88]. Accordingly, five more effective immunotherapy drugs (avelumab, atezolizumab, durvalumab, pembrolizumab, and nivolumab) and one targeted therapy (erdafitinib) have been approved. These drugs, adding to more accepted medications [89], could be used in a wide range of situations, from NMIBC to metastatic treatments, including adverse events management periods and maintenance interventions. But, these treatment breakthroughs, come at a high price for $\mathrm{BC}$ as one of the highest per-patient lifetime care costs. Concerns about quality and cost are particularly important in the diagnosis and treatment of BC. Particularly the urothelial carcinoma of the bladder has the most expensive lifetime therapy compared to other cancer with average costs of around $\$ 187,000 /$ case and a total treatment cost of $\mathrm{BC}$ is projected to be $\$ 4$ billion/year as reported by Zlatev et al. and the US Food and Drug Administration [90] [91]. Clinical management of $\mathrm{BC}$ varies widely among providers, raising concerns that compliance with care recommendations should be improved to enhance patient outcomes. Adding to that, these extremely high costs were estimated when the majority of BC therapies were substantially less expensive than they are now. Adding to their noticeable high monthly costs, drugs are often used for much longer periods of time.

\section{Discussion}

The public's response to the social gradient of premature mortality must be based on a deep knowledge and focused on a detailed understanding of the processes that underpin societal health disparities. Differences in BC mortality per type are the result differences in incidence and lethality. The factors that trigger SE incidence or survival inequalities are widely different. Therefore, it is crucial, to be able to study them separately according to the BC stage. Nowadays, $\mathrm{BC}$ data registries made it possible, using representative large samples of the overall population.

The factors that impact the SE environment are manifold and are influenced 
by a patient's economy, cultural, and social resources. Even if the intellectual and educational status, earnings, or professional situation seem to be good indicators of this setting, they are unable to summarize it. Moreover, a growing number of studies emphasize the importance of SE contextual factors within the patient's life ecosystem. As a result, synthetic indices are increasingly being used in works, allowing for an integrative study of the impact of patients' SE environment on their health as long as the statistical models used are tailored to the structure of the data.

A growing focus has been placed on the importance of health care by enhancing the quality or outcomes of treatment while lowering costs. BC management and care are expensive, but some areas of change may have a significant effect on outcomes and costs. Cost-cutting methods, such as using a cost-effective hybrid-technique rather than one simple expensive intervention, will help reduce costs without compromising results. Moreover, quality improvement practices in the management of NMIBC and MIBC by improving compliance with guideline concordant treatment, will improve outcomes, and avoid excessive costs. Will reduce possible side effects occurrence incidence and increase effectiveness rates.

Within all these factors, proactivity helps in preventing the potential harmful impacts especially with the COVID-19 global pandemic crisis. This acute health outbreak progressed randomly to be both health crisis and a serious wide-reaching global financial and economic concern. According to the International Monetary Fund (IMF), a global slump and a financial collapse are expected to happen starting from 2021. Thus, the frequency of major poverty might grow by $0.7 \%$ [92]. Yet, the cancer care of patients whose treatment was postponed must be fulfilled. Concerning low-income occupations, the economic consequences of COVID-19 will have a disproportionately large impact. Hence, losing a job is losing health insurance. According to Elkadhi et al., the two-year employment rate is over $12 \%$ less in Tunisia since the pandemic hit and this empowered the SE deprivation status which had a negative impact on healthcare services [93] [94]. It is important to deliver the right care at the right time for patients with cancer complications, even if they have loss of insurance coverage as supported by alternative federal or organizational funds.

Telemedicine was quickly introduced within the global health crisis with visits in patients being deferred or converted to distant virtual appointments. Nevertheless, Telemedicine is not uniformly accessible, and follow-ups can be interrupted to patients without internet connection or technological background. Involving health care providers, community relations are crucial to undertake strong follow-up actions. Anxiety and worries exacerbate the cancer results. Thus, managing patients' psychological effects is of big importance to reduce the pandemic's emotional impact, particularly for patients who are not able to express their worries when treating cancer.

Health behaviors describe an important portion of social disparities in mor- 
tality, indicating the significance of considering the continuous transformation in health behaviors while investigating their impact on social disparities.

Social determinants of health strongly influence an individual's life expectancy. Lower SE status and insurances were associated with a greater risk of mortality, while rural residence was a weak factor. Male sex workers were associated with both higher overall mortality and bladder cancer-specific mortality. Moreover, the implementation of public health programs could assist close the mortality gap in the treatment of muscle-invasive bladder cancer for these at-risk populations.

\section{Conclusions}

The size and profile of cancer incidence, mortality, and prevalence that will emerge over the next decades will be driven by ongoing shifts in population structure, the conditions in which people live, and the degree to which health systems can provide successful cancer prevention strategies.

$\mathrm{BC}$ patients following regular protocol of treatment are different in quarantine adherence amid epidemics of infectious diseases. Acceptance of pandemic outbreaks and quarantine rely on psychological, practical, and SE reasons. When quarantine is deemed to be necessary, public health officials should take steps to minimize the risk of non-compliance by establishing timely, clear quarantine rationales and information on protocols especially for cancer patients within the high deprived quintiles. The stress should be placed on social standards in order to encourage this altruist behavior.

In all genders, for patients with $\mathrm{BC}$, the $\mathrm{SE}$ status was an important indicative predictor of the overall survival and mortality estimations. The available studies focusing on the impact of poverty and SE status on BC treatment outcomes, or the overall survival still limited. But, in the wave of the COVID-19 outbreak, these studies have been accelerated, especially those investigating this parameter within all types of cancer. This pandemic spread worsened the status of all quintiles and added a pressure on the most deprived areas to register worrying numbers in mortality rates. Estimations were more serious for BC patients. Moreover, $\mathrm{BC}$ is the unique frequent disease that has a less pronounced prevalence for females than males. Although, recent data indicate that the high women mortality rates can be limited to those in more disadvantaged quintiles to discover poor survival rates.

The overall $\mathrm{BC}$ survival has improved more in wealthier patients in poorer communities, despite the management attempts of the widespread mortality discrepancies across the rich and the poor. More investigations are needed to explore and rectify these discrepancies between the affluent and the most deprived areas in BC survival.

Further research into the interaction between the hypothesized mediators and SES is needed to acquire a better understanding of the relationship between SES, treatment outcomes and survival that has been discovered in various observa- 
tional studies. Furthermore, modifiable factors underscore the significance of standardized clinical care for all bladder cancer patients across SES levels.

\section{Conflicts of Interest}

The authors declare no conflicts of interest.

\section{References}

[1] Bray, F., Ferlay, J., Soerjomataram, I., Siegel, R.L., Torre, L.A. and Jemal, A. (2018) Global Cancer Statistics 2018: GLOBOCAN Estimates of Incidence and Mortality Worldwide for 36 Cancers in 185 Countries. CA: A Cancer Journal for Clinicians, 68, 394-424. https://doi.org/10.3322/caac. 21492

[2] Ferlay, J., Ervik, M., Lam, F., Colombet, M., Mery, L., Piñeros, M., Znaor, A., Soerjomataram, I. and Bray, F. (2018) Global Cancer Observatory: Cancer Today. International Agency for Research on Cancer, Lyon.

[3] Silvestri, G.A., Jemal, A., Yabroff, K.R., Fedewa, S. and Sineshaw, H. (2021) Cancer Outcomes among Medicare Beneficiaries and Their Younger Uninsured Counterparts: Study Compares Cancer Outcomes among Medicare Beneficiaries and Their Younger Uninsured Counterparts. Health Affairs, 40, 754-762. https://doi.org/10.1377/hlthaff.2020.01839

[4] Erhunmwunsee, L., Seewaldt, V.L., Rebbeck, T.R. and Winn, R.A. (2021) From COVID-19 to Cancer, Watching Social Determinants Decide Life: When Will We Stop Spectating? Journal of the National Medical Association, 113, 436-439. https://doi.org/10.1016/j.jnma.2021.02.003

[5] Spiess, P.E., Agarwal, N., Bangs, R., Boorjian, S.A., Buyyounouski, M.K., Clark, P.E. and Gurski, L.A. (2017) Bladder Cancer, Version 5.2017, NCCN Clinical Practice Guidelines in Oncology. Journal of the National Comprehensive Cancer Network, 15, 1240-1267. https://doi.org/10.6004/jnccn.2017.0156

[6] Barki, C., Rahmouni, H.B. and Labidi, S. (2021) Prediction of Bladder Cancer Treatment Side Effects Using an Ontology-Based Reasoning for Enhanced Patient Health Safety. Informatics, 8, Article No. 55. https://doi.org/10.3390/informatics8030055

[7] Wang, T., Liu, S., Joseph, T. and Lyou, Y. (2020) Managing Bladder Cancer Care during the COVID-19 Pandemic Using a Team-Based Approach. Journal of Clinical Medicine, 9, Article No. 1574. https://doi.org/10.3390/jcm9051574

[8] Connor, M. J., Winkler, M., Miah, S., et al. (2020) COVID-19 Pandemic-Is Virtual Urology Clinic the Answer to Keeping the Cancer Pathway Moving? BJU International, 125, E3-E4. https://doi.org/10.1111/bju.15061

[9] Hastings, K.G., Boothroyd, D.B., Kapphahn, K., Hu, J., Rehkopf, D.H., Cullen, M. R. and Palaniappan, L. (2018) Socioeconomic Differences in the Epidemiologic Transition from Heart Disease to Cancer as the Leading Cause of Death in the United States, 2003 to 2015: An Observational Study. Annals of Internal Medicine, 169, 836-844. https://doi.org/10.7326/M17-0796

[10] Rodriguez-Acevedo, A. J., Green, A. C., Sinclair, C., van Deventer, E. and Gordon, L. G. (2020) Indoor Tanning Prevalence after the International Agency for Research on Cancer statement on Carcinogenicity of Artificial Tanning Devices: Systematic Review and Meta-Analysis. British Journal of Dermatology, 182, 849-859. https://doi.org/10.1111/bjd.18412

[11] Sylvester R.J., van der Meijden A.P.M., Oosterlinck W., Witjes J.A., Bouffioux C., 
Denis L., Newling D.W.W. and Kurth K. (2006) Predicting Recurrence and Progression in Individual Patients with Stage Ta T1 Bladder Cancer Using EORTC Risk Tables: A Combined Analysis of 2596 Patients from Seven EORTC Trials. European Urology, 49, 466-477. https://doi.org/10.1016/j.eururo.2005.12.031

[12] Ferlay, J., Colombet, M., Soerjomataram, I., Parkin, D. M., Piñeros, M., Znaor, A and Bray, F. (2021) Cancer Statistics for the Year 2020: An Overview. International Journal of Cancer, 149, 778-789. https://doi.org/10.1002/ijc.33588

[13] Okuyama, A. and Hori, M. (2021) Age-Specific Bladder Cancer Incidence Rate in the World. Japanese Journal of Clinical Oncology, 51, 511-512. https://doi.org/10.1093/jjco/hyab011

[14] Teoh, J.Y.C., Huang, J., Ko, W.Y.K., Lok, V., Choi, P., Ng, C.F. and Wong, M.C.S. (2020) Global Trends of Bladder Cancer Incidence and Mortality, and Their Associations with Tobacco Use and Gross Domestic Product Per Capita. European Urology, 78, 893-906. https://doi.org/10.1016/j.eururo.2020.09.006

[15] Chang, N.T., Chang, Y.H., Huang, Y.T. and Chen, S.C. (2021) Factors Associated with Refusal or Discontinuation of Treatment in Patients with Bladder Cancer: A Cohort Population-Based Study in Taiwan. International Journal of Environmental Research and Public Health, 18, Article No. 618. https://doi.org/10.3390/ijerph18020618

[16] Hamann, M.I., León-Règagnon, V., Fernández, M.V. and González, C.E. (2020) First Record of the Genus Choledocystus (Trematoda: Plagiorchiidae) for Amphibians of the Chacoan Region in Argentina. Anais da Academia Brasileira de Ciências, 92, Article ID: e20180384. https://doi.org/10.1590/0001-3765202020180384

[17] Ahmadi, M., Ranjbaran, H., Amiri, M.M., Nozari, J., Mirzajani, M.R., Azadbakht, M. and Hosseinimehr, S.J. (2012) Epidemiologic and Socioeconomic Status of Bladder Cancer in Mazandaran Province, Northern Iran. Asian Pacific Journal of Cancer Prevention, 13, 5053-5056. https://doi.org/10.7314/APJCP.2012.13.10.5053

[18] Shackley, D.C. and Clarke, N.W. (2005) Impact of Socioeconomic Status on Bladder Cancer Outcome. Current Opinion in Urology, 15, 328-331. https://doi.org/10.1097/01.mou.0000174965.26439.d1

[19] Yee, D.S., Ishill, N.M., Lowrance, W.T., Herr, H.W. and Elkin, E.B. (2011) Ethnic Differences in Bladder Cancer Survival. Urology, 78, 544-549.

https://doi.org/10.1016/j.urology.2011.02.042

[20] Haque, W., Lewis, G.D., Verma, V., Darcourt, J.G., Butler, E.B. and Teh, B.S. (2018) The Role of Adjuvant Chemotherapy in Locally Advanced Bladder Cancer. Acta Oncologica, 57, 509-515. https://doi.org/10.1080/0284186X.2017.1415461

[21] Trawalter, S., Hoffman, K. and Palmer, L. (2020) Out of Place: Socioeconomic Status, Use of Public Space, and Belonging in Higher Education. Journal of Personality and Social Psychology, 120, 131-144. https://doi.org/10.1037/pspi0000248

[22] Little, C., Alsen, M., Barlow, J., Naymagon, L., Tremblay, D., Genden, E. and van Gerwen, M. (2021) The Impact of Socioeconomic Status on the Clinical Outcomes of COVID-19; A Retrospective Cohort Study. Journal of Community Health, 46, 794-802. https://doi.org/10.1007/s10900-020-00944-3

[23] Vinitha Vijayan, D., Puranik, M.P. and Sowmya, K.R. (2020) Social Deprivation and Oral Health: A Narrative Review. International Journal of Applied Dental Sciences, 6, 355-360. https://doi.org/10.22271/oral.2020.v6.i4f.1087

[24] Townsend, P. (2020) 11 Objective and Subjective Deprivation. In: Townsend, P., Ed., Poverty in the United Kingdom, University of California Press, Berkeley, 
413-431.

[25] Patterson, R., Panter, J., Vamos, E.P., Cummins, S., Millett, C. and Laverty, A.A. (2020) Associations between Commute Mode and Cardiovascular Disease, Cancer, and All-Cause Mortality, and Cancer Incidence, Using Linked Census Data over 25 Years in England and Wales: A Cohort Study. The Lancet Planetary Health, 4, e186-e194. https://doi.org/10.1016/S2542-5196(20)30079-6

[26] Karadimitriou, N., Maloutas, T. and Arapoglou, V.P. (2021) Multiple Deprivation and Urban Development in Athens, Greece: Spatial Trends and the Role of Access to Housing. Land, 10, Article No. 290. https://doi.org/10.3390/land10030290

[27] Launoy, G., Launay, L., Bryère, J., Dejardin, O. and Guillaume, E. (2021) The European Deprivation Index: A Tool to Help Build an Evidence-Based Cancer Policy for Europe. In: Launoy, G., Zadnik, V. and Coleman, M.P., Eds., Social Environment and Cancer in Europe, Springer, Cham, 13-19.

https://doi.org/10.1007/978-3-030-69329-9 3

[28] Davis, L.E., Coburn, N.G., Hallet, J., Earle, C.C., Liu, Y., Myrehaug, S. and Mahar, A.L. (2020) Material Deprivation and Access to Cancer Care in a Universal Health Care System. Cancer, 126, 4545-4552. https://doi.org/10.1002/cncr.33107

[29] Mata, M.D.S. and Costa, Í.D.C.C. (2020) Composition of the Health Inequality Index Analyzed from the Inequalities in Mortality and Socioeconomic Conditions in a Brazilian State Capital. Ciência \& Saúde Coletiva, 25, 1629-1640. https://doi.org/10.1590/1413-81232020255.33312019

[30] De Boniface, J., Szulkin, R. and Johansson, A.L. (2021) Survival after Breast Conservation vs Mastectomy Adjusted for Comorbidity and Socioeconomic Status: A Swedish National 6-Year Follow-Up of 48,986 Women. JAMA Surgery, 156, 628-637. https://doi.org/10.1001/jamasurg.2021.1438

[31] Henstenburg, J.M., Lieber, A.M., Boniello, A.J., Kerbel, Y.E. and Shah, M. (2020) Higher Complication Rates after Management of Lower Extremity Fractures in Lower Socioeconomic Classes: Are Risk Adjustment Models Necessary? Trauma. https://doi.org/10.1177\%2F1460408620975693

[32] Wang, A.L., Zhong, X. and Hurd, Y. (2020) Comorbidity and Sociodemographic Determinants in COVID-19 Mortality in an US Urban Healthcare System. medRxiv. (Preprint) https://doi.org/10.1101/2020.06.11.20128926

[33] Abdel-Rahman, O. (2020) Prognostic Impact of Socioeconomic Status among Patients with Malignant Melanoma of the Skin: A Population-Based Study. Journal of Dermatological Treatment, 31, 571-575. https://doi.org/10.1080/09546634.2019.1657223

[34] Kou, K., Dasgupta, P., Aitken, J.F. and Baade, P.D. (2020) Impact of Area-Level Socioeconomic Status and Accessibility to Treatment on Life Expectancy after a Cancer Diagnosis in Queensland, Australia. Cancer Epidemiology, 69, Article ID: 101803. https://doi.org/10.1016/j.canep.2020.101803

[35] Kuhn, Y., Koscielny, A., Glowka, T., Hirner, A., Kalff, J.C. and Standop, J. (2010) Postresection Survival Outcomes of Pancreatic Cancer According to Demographic Factors and Socio-Economic Status. European Journal of Surgical Oncology, 36, 496-500. https://doi.org/10.1016/j.ejso.2009.08.006

[36] Russell, B., Moss, C., Papa, S., Irshad, S., Ross, P., Spicer, J. and Van Hemelrijck, M. (2020) Factors Affecting COVID-19 Outcomes in Cancer Patients: A First Report from Guy's Cancer Center in London. Frontiers in Oncology, 10, Article No. 1279. https://doi.org/10.3389/fonc.2020.01279

[37] Luo, J., Kroenke, C.H., Hendryx, M., Shadyab, A.H., Liu, N., Chen, X. and Wac- 
tawski-Wende, J. (2021) Mediation Analysis of Racial Disparities in Triple-Negative Breast Cancer Incidence among Postmenopausal Women. Breast Cancer Research and Treatment, 188, 283-293. https://doi.org/10.1007/s10549-021-06158-y

[38] Richiardi, L., Bellocco, R. and Zugna, D. (2013) Mediation Analysis in Epidemiology: Methods, Interpretation and Bias. International Journal of Epidemiology, 42, 1511-1519. https://doi.org/10.1093/ije/dyt127

[39] Barki, C., Labidi, S. and Rahmouni, H.B. (2017) Ontology-Driven Generation of Radiation Protection Procedures. International Journal of Environmental, Chemical, Ecological, Geological and Geophysical Engineering, 11, 256-261.

[40] Bryere, J., Dejardin, O., Launay, L., Colonna, M., Grosclaude, P. and Launoy, G. (2018) Socioeconomic Status and Site-Specific Cancer Incidence, a Bayesian Approach in a French Cancer Registries Network Study. European Journal of Cancer Prevention, 27, 391-398. https://doi.org/10.1097/CEJ.0000000000000326

[41] Bryere, J., Tron, L., Menvielle, G. and Launoy, G. (2019) The Respective Parts of Incidence and Lethality in Socioeconomic Differences in Cancer Mortality. An Analysis of the French Network Cancer Registries (FRANCIM) Data. International Journal for Equity in Health, 18, Article No. 189. https://doi.org/10.1186/s12939-019-1087-y

[42] Guy, L. (2019) Impact de l'intégration du test immunologique dans le programme de dépistage du cancer colorectal en France. Bulletin du Cancer, 106, 703-706. https://doi.org/10.1016/j.bulcan.2019.01.011

[43] Fauvernier, M., Roche, L., Uhry, Z., Tron, L., Bossard, N. and Remontet, L. (2019) Challenges in the Estimation of Net Survival Working Survival Group. Multi-Dimensional Penalized Hazard Model with Continuous Covariates: Applications for Studying Trends and Social Inequalities in Cancer Survival. Journal of the Royal Statistical Society: Series C (Applied Statistics), 68, 1233-1257. https://doi.org/10.1111/rssc. 12368

[44] Assari, S., Khoshpouri, P. and Chalian, H. (2019) Combined Effects of Race and Socioeconomic Status on Cancer Beliefs, Cognitions, and Emotions. Healthcare, 7, Article No. 17. https://doi.org/10.3390/healthcare7010017

[45] Kweon, S.S., Kim, M.G., Kang, M.R., Shin, M.H. and Choi, J.S. (2017) Diference of Stage at Cancer Diagnosis by Socioeconomic Status for Four Target Cancers of the National Cancer Screening Program in Korea: Results from the Gwangju and Jeonnam Cancer Registries. Journal of Epidemiology, 27, 299-304. https://doi.org/10.1016/j.je.2016.07.004

[46] Sung, J.M., Martin, J.W., Jefferson, F.A., Sidhom, D.A., Piranviseh, K., Huang, M. and Youssef, R.F. (2019) Racial and Socioeconomic Disparities in Bladder Cancer Survival: Analysis of the California Cancer Registry. Clinical Genitourinary Cancer, 17, 995-1002. https://doi.org/10.1016/j.clgc.2019.05.008

[47] Mihor, A., Tomsic, S., Zagar, T., Lokar, K. and Zadnik, V. (2020) Socioeconomic Inequalities in Cancer Incidence in Europe: A Comprehensive Review of Population-Based Epidemiological Studies. Radiology and Oncology, 54, 1-13. https://doi.org/10.2478/raon-2020-0008

[48] Golombos, D.M., O’Malley, P., Lewicki, P., Nguyen, D.P., Stone, B.V., Al Awamlh, B.A.H. and Scherr, D.S. (2017) The Impact of Socioeconomic Status on Perioperative Complications and Oncologic Outcomes in Patients Undergoing Radical Cystectomy. World Journal of Urology, 35, 1063-1071. https://doi.org/10.1007/s00345-016-1972-6

[49] Barki, C., Rahmouni, H.B. and Labidi, S. (2021) Model-Based Prediction of Onco- 
therapy Risks and Side Effects in Bladder Cancer. Procedia Computer Science, 181, 818-826. https://doi.org/10.1016/j.procs.2021.01.235

[50] Lee, H.E., Zaitsu, M., Kim, E.A. and Kawachi, I. (2020) Occupational Class and Cancer Survival in Korean Men: Follow-Up Study of Nation-Wide Working Population. International Journal of Environmental Research and Public Health, 17, Article No. 303. https://doi.org/10.3390/ijerph17010303

[51] Stringhini, S., Sabia, S., Shipley, M., Brunner, E., Nabi, H., Kivimaki, M. and Singh-Manoux, A. (2010) Association of Socioeconomic Position with Health Behaviors and Mortality. JAMA, 303, 1159-1166. https://doi.org/10.1001/jama.2010.297

[52] Mason, S.J., Downing, A., Wright, P., Hounsome, L., Bottomley, S.E., Corner, J. and Glaser, A.W. (2018) Health-Related Quality of Life after Treatment for Bladder Cancer in England. British Journal of Cancer, 118, 1518-1528.

https://doi.org/10.1038/s41416-018-0084-Z

[53] Wong, M.C., Fung, F.D., Leung, C., Cheung, W.W., Goggins, W.B. and Ng, C.F. (2018) The Global Epidemiology of Bladder Cancer: A Joinpoint Regression Analysis of Its Incidence and Mortality Trends and Projection. Scientific Reports, 8, Article No. 1129. https://doi.org/10.1038/s41598-018-19199-Z

[54] Afshar, N., English, D.R., Blakely, T., Thursfield, V., Farrugia, H., Giles, G.G. and Milne, R.L. (2020) Differences in Cancer Survival by Area-Level Socio-Economic Disadvantage: A Population-Based Study Using Cancer Registry Data. PLoS ONE, 15, Article ID: 0228551. https://doi.org/10.1371/journal.pone.0228551

[55] Cette, G., Lopez, J., Mairesse, J. and Nicoletti, G. (2020) Economic Adjustment during the Great Recession: The Role of Managerial Quality. Working Paper No. w27954, National Bureau of Economic Research, Cambridge. https://doi.org/10.3386/w27954

[56] Mackenbach, J.P., Hu, Y., Artnik, B., Bopp, M., Costa, G., Kalediene, R. and Nusselder, W.J. (2017) Trends in inequalities in mortality amenable to health care in 17 European countries. Health Affairs, 36, 1110-1118. https://doi.org/10.1377/hlthaff.2016.1674

[57] Singh, G.K., Kogan, M.D. and Slifkin, R.T. (2017) Widening Disparities in Infant Mortality and Life Expectancy between Appalachia and the Rest of the United States, 1990-2013. Health Affairs, 36, 1423-1432. https://doi.org/10.1377/hlthaff.2016.1571

[58] Dickman, S.L., Himmelstein, D.U. and Woolhandler, S. (2017) Inequality and the health-Care System in the USA. The Lancet, 389, 1431-1441. https://doi.org/10.1016/S0140-6736(17)30398-7

[59] Dutta, A. and Bandyopadhyay, S. (2019) Policy Intervention for Access to Medicine: Does It Work Similarly for Poor and Non-Poor? The International Journal of Health Planning and Management, 34, e557-e568. https://doi.org/10.1002/hpm.2671

[60] Hansen, H., Schäfer, I., Porzelt, S., Kazek, A., Lühmann, D. and Scherer, M. (2020) Regional and Patient-Related Factors Influencing the Willingness to Use General Practitioners as Coordinators of the Treatment in Northern Germany-Results of a Cross-Sectional Observational Study. BMC Family Practice, 21, Article No. 110. https://doi.org/10.1186/s12875-020-01180-3

[61] Singh, G.K. and Jemal, A. (2018) Socioeconomic Inequalities in Cancer Incidence and Mortality. In: The American Cancer Society, Ed., The American Cancer Society's Principles of Oncology. Prevention to Survivorship, Wiley, Hoboken, 23-32. https://doi.org/10.1002/9781119468868.ch3 
[62] Feinglass, J., Wang, J.A., Ye, J., Tessier, R. and Kim, H. (2021) Hospital Care for Opioid use in Illinois, 2016-2019. The Journal of Behavioral Health Services \& Research, 1-13. https://doi.org/10.1007/s11414-020-09748-8

[63] Vanthomme, K., Vandenheede, H. and Hagedoorn, P. (2016) Socioeconomic Disparities in Lung Cancer Mortality in Belgian Men and Women (2001-2011): Does It Matter Who You Live with? BMC Public Health, 16, Article No. 493. https://doi.org/10.1186/s12889-016-3139-1

[64] Siegel, R.L., Miller, K.D. and Jemal, A. (2020) Cancer Statistics, 2020. CA: A Cancer Journal for Clinicians, 70, 7-30. https://doi.org/10.3322/caac.21590

[65] Siegel, R.L., Jemal, A., Wender, R.C., Gansler, T., Ma, J. and Brawley, O.W. (2018) An Assessment of Progress in Cancer Control. CA: A Cancer Journal for Clinicians, 68, 329-339. https://doi.org/10.3322/caac. 21460

[66] Kehm, R.D., Spector, L.G., Poynter, J.N., Vock, D.M., Altekruse, S.F. and Osypuk, T.L. (2018) Does Socioeconomic Status Account for Racial and Ethnic Disparities in Childhood Cancer Survival? Cancer, 124, 4090-4097. https://doi.org/10.1002/cncr.31560

[67] Withrow, D.R., Berrington de González, A., Spillane, S., Freedman, N.D., Best, A.F., Chen, Y. and Shiels, M.S. (2019) Trends in Mortality Due to Cancer in the United States by Age and County-Level Income, 1999-2015. JNCI: Journal of the National Cancer Institute, 111, 863-866. https://doi.org/10.1093/jnci/djz123

[68] Cao, W., Chen, H.D., Yu, Y.W., Li, N. and Chen, W.Q. (2021) Changing Profiles of Cancer Burden Worldwide and in China: A Secondary Analysis of the Global Cancer Statistics 2020. Chinese Medical Journal, 134, 783-791. https://doi.org/10.1097/CM9.0000000000001474

[69] Sung, H., Ferlay, J., Siegel, R.L., Laversanne, M., Soerjomataram, I., Jemal, A. and Bray, F. (2021) Global Cancer Statistics 2020: GLOBOCAN Estimates of Incidence and Mortality Worldwide for 36 Cancers in 185 Countries. CA: A Cancer Journal for Clinician, 71, 209-249. https://doi.org/10.3322/caac.21660

[70] Lortet-Tieulent, J., Georges, D., Bray, F. and Vaccarella, S. (2020) Profiling Global Cancer Incidence and Mortality by Socioeconomic Development. International Journal of Cancer, 147, 3029-3036. https://doi.org/10.1002/ijc.33114

[71] Alexeeff, S.E., Schaefer, C.A., Kvale, M.N., Shan, J., Blackburn, E.H., Risch, N. and Van Den Eeden, S.K. (2019) Telomere Length and Socioeconomic Status at Neighborhood and Individual Levels among 80,000 Adults in the Genetic Epidemiology Research on Adult Health and Aging cohort. Environmental Epidemiology, 3, Article No. e049. https://doi.org/10.1097/EE9.0000000000000049

[72] Khan, R.J., Needham, B.L., Advani, S., Brown, K., Dagnall, C., Xu, R. and Davis, S.K. (2021) Association of Childhood Socioeconomic Status with Leukocyte Telomere Length among African Americans and the Mediating Role of Behavioral and Psychosocial Factors: Results from the GENE-FORECAST Study. Journal of Racial and Ethnic Health Disparities, 1-12. https://doi.org/10.1007/s40615-021-01040-5

[73] Schmidt, A.L., Bakouny, Z., Bhalla, S., Steinharter, J.A., Tremblay, D.A., Awad, M.M. and Doroshow, D.B. (2020) Cancer Care Disparities during the COVID-19 Pandemic: COVID-19 and Cancer Outcomes Study. Cancer Cell, 38, 769-770. https://doi.org/10.1016/j.ccell.2020.10.023

[74] Saini, K.S., Tagliamento, M., Lambertini, M., McNally, R., Romano, M., Leone, M. and de Azambuja, E. (2020) Mortality in Patients with Cancer and Coronavirus Disease 2019: A Systematic Review and Pooled Analysis of 52 Studies. European Journal of Cancer, 139, 43-50. https://doi.org/10.1016/j.ejca.2020.08.011 
[75] Zaniboni, A., Ghidini, M., Grossi, F., Indini, A., Trevisan, F., Iaculli, A. and Petrelli, F. (2020) A Review of Clinical Practice Guidelines and Treatment Recommendations for Cancer Care in the COVID-19 Pandemic. Cancers, 12, Article No. 2452. https://doi.org/10.3390/cancers12092452

[76] Singh, A.K., Singh, R., Joshi, S.R. and Misra, A. (2021) Mucormycosis in COVID-19: A Systematic Review of Cases Reported Worldwide and in India. Diabetes \& Metabolic Syndrome. Clinical Research \& Reviews, 15, Article ID: 102146. https://doi.org/10.1016/j.dsx.2021.05.019

[77] Williams, H., Hutchinson, D. and Stone, H. (2021) Watching Brief: The Evolution and Impact of COVID-19 Variants B.1.1.7, B.1.351, P.1 and B.1.617. Global Biosecurity, 3, 1-127.

[78] Ahmadikia, K., Hashemi, S.J., Khodavaisy, S., Getso, M.I., Alijani, N., Badali, H. and Rezaie, S. (2021) The Double-Edged Sword of Systemic Corticosteroid Therapy in Viral Pneumonia: A Case Report and Comparative Review of Influenza-Associated Mucormycosis versus COVID-19 Associated Mucormycosis. Mycoses, 64, 798-808. https://doi.org/10.1111/myc.13256

[79] Rao, V., Arakeri, G., Subash, A., Ajaikumar, B.S., Patil, R., Hale, B. and Mendes, R.A. (2021) Decreased Cancer Consultations in the COVID-19 Era: A Concern for Delay in Early Cancer Diagnosis in India. JCO Global Oncology, 7, 408-409.

https://doi.org/10.1200/GO.21.00030

[80] Dai, M., Liu, D., Liu, M., Zhou, F., Li, G., Chen, Z. and Cai, H. (2020) Patients with Cancer Appear More Vulnerable to SARS-CoV-2: A Multicenter Study during the COVID-19 Outbreak. Cancer Discovery, 10, 783-791. https://doi.org/10.1158/2159-8290.CD-20-0422

[81] Gordon, N.P. and Crouch, E. (2019) Digital Information Technology Use and Patient Preferences for Internet-Based Health Education Modalities: Cross-Sectional Survey Study of Middle-Aged and Older Adults with Chronic Health Conditions. JMIR Aging, 2, Article No. e12243. https://doi.org/10.2196/12243

[82] Ware, P., Bartlett, S.J., Paré, G., Symeonidis, I., Tannenbaum, C., Bartlett, G. and Ahmed, S. (2017) Using eHealth Technologies: Interests, Preferences, and Concerns of Older Adults. Interactive Journal of Medical Research, 6, Article No. e3. https://doi.org/10.2196/ijmr.4447

[83] Russell, B., Hemelrijck, M.V., Gårdmark, T., Holmberg, L., Kumar, P., Bellavia, A. and Häggström, C. (2020) A Mediation Analysis to Explain Socio-Economic Differences in Bladder Cancer Survival. Cancer Medicine, 9, 7477-7487. https://doi.org/10.1002/cam4.3418

[84] Weiner, A.B., Keeter, M.K., Manjunath, A. and Meeks, J.J. (2018) Discrepancies in Staging, Treatment, and Delays to Treatment May Explain Disparities in Bladder Cancer Outcomes: An Update from the National Cancer Data Base (2004-2013). Urologic Oncology: Seminars and Original Investigations, 36, 237.e9-237.e17. https://doi.org/10.1016/j.urolonc.2017.12.015

[85] Golombos, D.M., O’Malley, P., Lewicki, P., Nguyen, D.P., Stone, B.V., Al Awamlh, B.A.H. and Scherr, D.S. (2017) The Impact of Socioeconomic Status on Perioperative Complications and Oncologic Outcomes in Patients Undergoing Radical Cystectomy. World Journal of Urology, 35, 1063-1071. https://doi.org/10.1007/s00345-016-1972-6

[86] Modi, P.K., Hollenbeck, B.K., Oerline, M., Weizer, A.Z., Montgomery, J.S., Kaffenberger, S.D. and Ellimoottil, C. (2019) Real-World Impact of Minimally Invasive versus Open Radical Cystectomy on Perioperative Outcomes and Spending. Urolo- 
gy, 125, 86-91. https://doi.org/10.1016/j.urology.2018.10.022

[87] Azadeh, N.A., Ghodoussipour, S., Pearce, S., Bhanvadia, S. and Daneshmand, S. (2019) Socioeconomic and Insurance Status Are Independent Prognostic Indicators of Higher Disease Stage and Worse Prognosis in Bladder Cancer.b Urologic Oncology: Seminars and Original Investigations, 37, 784-790. https://doi.org/10.1016/j.urolonc.2019.04.021

[88] Reis, L.O., Moro, J.C., Ribeiro, L.F., Voris, B.R. and Said, M.V. (2016) Are We Following the Guidelines on Non-Muscle Invasive Bladder Cancer? International braz $j$ urol, 42, 22-28. https://doi.org/10.1590/S1677-5538.IBJU.2015.0122

[89] Simon, S. (2019) FDA Approves Padcev (Enfortumab vedotin-ejfv) for Bladder Cancer. American Cancer Society, Atlanta.

[90] US Food and Drug Administration (2020) FDA Approves Pembrolizumab for BCG-Unresponsive, High-Risk Non-Muscle Invasive Bladder Cancer. US Food and Drug Administration, Silver Spring.

[91] Zlatev, D.V., Altobelli, E.L. and Joseph C. (2015) Advances in Imaging Technologies in the Evaluation of High-Grade Bladder Cancer. Urologic Clinics, 42, 147-157. https://doi.org/10.1016/j.ucl.2015.01.001

[92] Decerf, B., Ferreira, F.H., Mahler, D. and Sterck, O. (2020) Lives and Livelihoods: Estimates of the Global Mortality and Poverty Effects of the Covid-19 Pandemic. Policy Research Working Paper, 9277, 1-37. https://doi.org/10.1596/1813-9450-9277

[93] ElKadhi, Z., Elsabbagh, D., Frija, A., Lakoud, T., Wiebelt, M. and Breisinger, C. (2020) The Impact of Covid-19 on Tunisia's Economy, Agri-Food System, and Households. No. 5, International Food Policy Research Institute (IFPRI), Washington DC. https://doi.org/10.2499/p15738coll2.133737

[94] Mohamed, A. (2021) A Literature Review: The Impact of COVID-19 Pandemic on Somaliland Economy. Open Journal of Social Sciences, 9, 54-64.

https://doi.org/10.4236/jss.2021.92004 